\title{
Hypoglycemic myocardial stunning as cause of cardiogenic shock in a patient with ischemic cardiomyopathy: A case report and review of literature
}

\author{
Khawar Maqsood ${ }^{1^{*}}$, Ghazi Mirrani ${ }^{2}$, Nosheen Sarwar ${ }^{2}$, Amatur R. Amarah $^{1}$, \\ Muhammad Rizwan Sardar ${ }^{3}$, Timothy A. Shapiro ${ }^{3}$ \\ ${ }^{1}$ Department of Medicine, Baystate Medical Center, Tufts University School of Medicine, Springfield, USA; \\ *Corresponding Author: dockmaq@gmail.com \\ ${ }^{2}$ Department of Medicine, Division of Internal Medicine, Lankenau Medical Center, Wynnewood, USA \\ ${ }^{3}$ Department of Medicine, Division of Cardiology, Lankenau Medical Center, Wynnewood, USA
}

Received 27 January 2013; revised 26 February 2013; accepted 12 March 2013

\begin{abstract}
Hypoglycemia is a common complication seen in patients with diabetes mellitus and has been proven to have adverse effects on cardiovascular mortality. Hypoglycemia can potentially lead to worsening of cardiac function in patients with ischemic heart disease. We present a case of cardiogenic shock in a patient with hypoglycemia secondary to insulin accumulation due to worsening renal function with dramatic recovery of shock once his sugars normalized.
\end{abstract}

Keywords: Chronic Heart Failure; Hypoglycemia; Myocardial Stunning; Ischemic Cardiomyopathy; Cardiogenic Shock

\section{INTRODUCTION}

Chronic heart failure and diabetes are very common diseases and around $40 \%$ patients with CHF have DM [1]. Hypoglycemia has been independently associated with worse cardiovascular outcomes in diabetics [2]. Labile sugars with rapid fluctuations between high and low sugars have been proven to increase mortality in CHF [3]. Hypoglycemia has been associated with ischemic events in patients with coronary artery disease (CAD). It is proposed that hypoglycemia and rapid changes in blood glucose levels lead to increase catecholamines release which results in vasoconstriction, platelet aggregation and ischemia [4,5]. We report a case of reversible cardiogenic shock in a patient with hypoglycemia secondary to insulin accumulation. We have theorized a novel mechanism for hypoglycemic myocardial stunning in our patient.

\section{CASE PRESENTATION}

A 58-year-old African-American male presented to our institution with a 2 day history of confusion and visual hallucinations. He stated that he was feeling weak, was diaphoretic and tired for almost a week and was unable to check his sugars because he ran out of his testing strips. He thought his sugars were low and he was drinking orange juice every time he felt weak or tired. On the day of admission he had a near-syncopal episode and his wife found him on the floor and brought him to the emergency department. Of note, he drank 2 glasses of orange juice before coming to the emergency department. He denied any chest pain, loss of consciousness or loss of bladder/bowel control but did complain of ongoing dyspnea on exertion and orthopnea for a week for which his primary care physician increased his furosemide dose from $80 \mathrm{mg}$ PO daily to $160 \mathrm{mg}$ PO daily one day before admission.

His past medical history was significant for ischemic cardiomyopathy. He was admitted with congestive heart failure and hypoglycemia one month ago and a transthoracic echocardiogram performed at that time revealed an ejection fraction of $35 \%$ to $40 \%$, inferolateral akinesis and moderate to severe mitral regurgitation. He was admitted with a non-ST elevation myocardial infarction 5 months ago and his coronary angiography at that time showed a patent mid-LAD stent, $80 \%$ apical LAD stenosis, occluded diagonal-1, $80 \%$ to $90 \%$ stenosis of small diagonal-2, a large left circumflex with mid vessel 50\% stenosis, occluded right coronary artery with left to right collaterals filling the posterior descending artery and a large obtuse marginal-1 with in-stent thrombosis. His obtuse marginal-1 was intervened on as the culprit lesion. 
He also had a history of hypertension, hyperlipidemia, chronic renal impairment and obstructive sleep apnea. His medications included 75 units of insulin glargine (Lantus) along with 65 units of Novolog 70/30, both of which he was taking in the morning. He was also on aspirin, prasugrel, carvedilol, lisinopril, atorvastatin, hydralazine and furosemide.

On examination, he had Cheyne-Stokes breathing and was delirious. His blood pressure was 105/60, heart rate 110 and regular and his oxygen saturations were $96 \%$ on ambient air. He was obese and neck veins were difficult to be assessed for jugular venous pressure. His lungs had faint crackles towards bases. Cardiac examination revealed a S3 gallop and a grade 3/6 holosystolic murmur heard at the apex. He had 2+ pedal edema and all his extremities were cold to touch suggesting a low flow state. His laboratory work up showed a hemoglobin of 13.4 g/dL, white blood cell count 4800/UL, potassium 5.5 $\mathrm{mEq} / \mathrm{L}$, BUN $49 \mathrm{mg} / \mathrm{dL}$, creatinine $3.5 \mathrm{mg} / \mathrm{dL}$ from his baseline $3.2 \mathrm{mg} / \mathrm{dL}$, BNP $1760 \mathrm{pg} / \mathrm{mL}$ and troponin 0.18 $\mathrm{ng} / \mathrm{mL}$ which peaked at $0.22 \mathrm{ng} / \mathrm{mL}$. His glucose initially was $95 \mathrm{mg} / \mathrm{dL}$. EKG showed sinus tachycardia with left bundle branch block (old) and premature ventricular complexes. His chest $\mathrm{x}$-ray showed signs of pulmonary congestion. A bedside echocardiogram was performed which showed an ejection fraction of $20 \%$ with global hypokinesis and severe mitral regurgitation.

A right heart catheterization was done which revealed a right atrial pressure of $21 \mathrm{~mm} \mathrm{Hg}, \mathrm{RV}$ systolic pressure of $74 \mathrm{~mm} \mathrm{Hg}$, RV diastolic pressure of $32 \mathrm{~mm} \mathrm{Hg}$, pulmonary artery systolic pressure of $61 \mathrm{~mm} \mathrm{Hg}$, pulmonary artery diastolic pressure of $39 \mathrm{~mm} \mathrm{Hg}$ with a mean pulmonary arterial pressure of $52 \mathrm{~mm} \mathrm{Hg}$ and a pulmonary capillary wedge pressure of $36 \mathrm{~mm} \mathrm{Hg}$. His mixed venous saturations were $46 \%$, arterial saturation $90 \%$, cardiac output by Fick principle was $3.7 \mathrm{~L} / \mathrm{min}$ and cardiac index was $1.5 \mathrm{~L} / \mathrm{m}^{2}$.

He was diagnosed as having cardiogenic shock and was transferred to ICU for further management. He was started on milrinone drip at $0.2 \mathrm{mcg} / \mathrm{kg} / \mathrm{min}$ and his carvedilol was held due to shock and borderline blood pressure. In the ICU, his mental status worsened after a few hours and his blood sugar was found to be $33 \mathrm{mg} / \mathrm{dL}$. He received dextrose-50\% and orange juice and his blood glucose was raised to $96 \mathrm{mg} / \mathrm{dL}$ with dramatic recovery in his mental status. His insulin was on hold since admission. Around 6 hours later, he had a similar episode and his blood sugar was found to be $40 \mathrm{mg} / \mathrm{dL}$ which was treated again with dextrose-50\%. His sugars were closely monitored with as needed supplementation and his point of care sugars stayed stable.

He had a dramatic improvement in his hemodynamics by day 2 when his blood sugars normalized. He was warm to touch and his mixed venous saturations were
78.3\% suggesting adequate cardiac output and normalization of systemic vascular resistance. At this stage he was weaned off the milrinone drip. His repeat echocardiogram, 48 hours after admission and off the milrinone drip, showed that his ejection fraction has improved to $25 \%$. His sugars were stabilized further in collaboration with an endocrinologist and his insulin was reintroduced. He was restarted on beta-blocker slowly and the dose was titrated up. Given his renal insufficiency, it was decided not to restart his insulin glargine. He was transferred to the telemetry floor and subsequently was discharged home in a week.

\section{DISCUSSION}

Hypoglycemia is associated with increased catecholamine release in the body which along with electrolyte abnormalities can lead to prolongation of cardiac repolarization and can result in arrhythmias [6]. Both type-1 and type-2 diabetics can have prolongation of QT intervals and arrhythmias in acute hypoglycemic events [7]. Typical EKG changes for ischemia have been seen during hypoglycemia in patients with CAD without DM [8]. Hypoglycemia can lead to an increased infarct size and this has been proven in animal and human studies [9-11]. Silent ischemia is the most common manifestation of myocardial ischemia and is more common in CAD with DM [12-14]. Takotsubo cardiomyopathy has been described in patients with anorexia nervosa and hypoglycemic coma and excessive catecholamines release seconddary to hypoglycemia has been proposed as the etiology [15]. All these findings suggest the adverse effects of hypoglycemia on cardiovascular system.

The heart has the capacity to use a variety of metabolic substrates for energy production and has the ability to rapidly adapt its substrate utilization in a situation with change in substrate supply. Fatty acids are the major metabolic substrate for the heart. Glucose along with lactate, ketones and amino acids contribute up to $30 \%$ of generated myocardial ATP. Glucose entry into cardiomyocytes is primarily through GLUT4 transporter which is dependent on insulin for its action. Glucose is not the major metabolic substrate in the heart at rest but assumes greater importance in conditions like ischemia; increased workload and pressure overload e.g. hypertrophy $[16,17]$. A study conducted on dogs showed that suppressed expression and translocation of myocardial GLUT4 and resulting reduced myocardial glucose uptake and utilization in ischemic conditions were clearly associated with ischemia-reperfusion myocardial injury [18]. GLUT4 translocation is different under different stimuli. This was proven by immunogold labeling of GLUT4 and its localization after 30 minutes exposure to insulin and ischemia. Insulin increased $\mathrm{T}$ tubule membrane GLUT4 but had 
less effect on sarcolemmal GLUT4. Ischemia, on the other hand, resulted in GLUT4 translocation to both, T tubule and sarcolemmal membranes [19]. A deficiency of GLUT4 has been associated with profound alterations in cardiomyocyte $\mathrm{Ca}^{+2}$ and $\mathrm{pH}$ homeostasis and can have adverse effects on excitation-contraction coupling in cardiomyocytes [20]. These studies signify the importance of glucose in cardiomyocytes specially under ischemic conditions.

Insulin is known to have a cardioprotective role [21] and elicits a positive inotropic effect on the heart. Insulin dependent positive inotropic effect (PIE) is mediated by a calcium-dependent PI-3 kinase-mediated pathway and a calcium independent pathway which involves GLUT4 and affirms the glucose dependency in inotropic response to insulin [22]. Diabetes mellitus is characterized by enhanced fatty acid metabolism and reduced glucose and lactate metabolism in the heart $[23,24]$ but insulin replacement results in increased glucose use and reversal of myocardial dysfunction in patients with diabetic cardiomyopathy [25-27].

This is the first case report of hypoglycemia-induced myocardial stunning. Our patient had ischemic cardiomyopathy and had significant underlying coronary artery disease. His presentation with acute decompensated congestive heart failure in the setting of hypoglycemia twice and his dramatic recovery once blood sugars were normalized point towards hypoglycemia as the etiology of his depressed cardiac function. Our patient did not have high enough troponins or new regional wall motion abnormalities to suggest coronary artery disease as the primary underlying etiology and his echocardiographic images were not consistent with Takotsubo cardiomyopathy.

We propose that probably due to ongoing silent ischemia and insulin toxicity, the cardiac metabolism was shifted towards glucose as the primary substrate and resulted in a stunning-like phenomenon due to hypoglycemia secondary to accumulation of excessive insulin. Our patient was on a $\beta$-blocker which might have masked the catecholamine induced symptoms of hypoglycemia. Further studies will be needed to clearly explain a cause and effect relationship, if any, between hypoglycemia and myocardial stunning.

\section{CONCLUSION}

CHF and DM are common diseases and hypoglycemia is a common complication in treated diabetics. Hypoglycemia can lead to worsening of myocardial function in patients with ischemic cardiomyopathy and silent ischemia. This is the first case report of hypoglycemic myocardial stunning and highlights the importance of close glucose monitoring and adequate glucose control in pa- tients with ischemic cardiomyopathy and CAD.

\section{REFERENCES}

[1] Adams Jr., K.F., Fonarow, G.C., Emerman, C.L., et al. (2005) ADHERE Scientific Advisory Committee and Investigators. Characteristics and outcomes of patients hospitalized for heart failure in the United States: Rationale, design, and preliminary observations from the first 100, 000 cases in the Acute Decompensated Heart Failure National Registry (ADHERE). American Heart Journal, 149, 209-216. doi:10.1016/j.ahj.2004.08.005

[2] Abraira, C., Colwell, J.A., Nuttall, F.Q., Sawin, C.T., Nagel, N.J., Comstock, J.P., Emanuele, N.V., Levin, S.R., Henderson, W. and Lee, H.S. (1995) Veterans Affairs Cooperative Study on glycemic control and complications in type II diabetes (VA CSDM): Results of the feasibility trial: Veterans Affairs Cooperative Study in Type II Diabetes. Diabetes Care, 18, 1113-1123.

doi:10.2337/diacare.18.8.1113

[3] Dungan, K.M., Binkley, P., Nagaraja, H.N., Schuster, D. and Osei, K. (2011) The effect of glycaemic control and glycaemic variability on mortality in patients hospitalized with congestive heart failure. Diabetes/Metabolism Research and Reviews, 27, 85-93. doi:10.1002/dmrr.1155

[4] DeFronzo, R.A., Hendler, R. and Christensen, N. (1980) Stimulation of counterregulatory hormonal responses in diabetic man by a fall in glucose concentration. Diabetes, 29, 125-131.

[5] Galassetti, P. and Davis, S.N. (2000) Effects of insulin per se on neuroendocrine and metabolic counter-regulatory responses to hypoglycaemia. Clinical Science (London), 99, 351-362. doi:10.1042/CS20000022

[6] Heller, S.R. (2002) Abnormalities of the electrocardiogram during hypoglycaemia: The cause of the dead in bed syndrome? International Journal of Clinical Practice, S129, 27-322.

[7] Marques, J.L., George, E., Peacey, S.R., Harris, N.D., Macdonald, I.A., Cochrane, T. and Heller, S.R. (1997) Altered ventricular repolarization during hypoglycaemia in patients with diabetes. Diabetic Medicine, 14, 648-654. doi:10.1002/(SICI)1096-9136(199708)14:8<648::AID-DI A418>3.0.CO;2-1

[8] Koh, H., Nambu, S., Tsushima, M., Nishioheda, Y., Murakami, K. and Ikeda, M. (1984) The effects of insulin on the cardiovascular system in patients with coronary heart disease. Arzneimittelforschung, 34, 185-190.

[9] Libby, P., Maroko, P.R. and Braunwald, E. (1975) The effect of hypoglycemia on myocardial ischemic injury during acute experimental coronary artery occlusion. Circulation, 51, 621-626. doi:10.1161/01.CIR.51.4.621

[10] Bansal, S., Toh, S.H. and LaBresh, K.A. (1983) Chest pain as a presentation of reactive hypoglycemia. Chest, 84, 641-642. doi:10.1378/chest.84.5.641

[11] Pladziewicz, D.S. and Nesto, R.W. (1989) Hypoglycemia-induced silent myocardial ischemia. American Journal of Cardiology, 63, 1531-1532. doi:10.1016/0002-9149(89)90025-8 
[12] Nesto, R.W., Phillips, R.T., Kett, K.G., Hill, T., Perper, E., Young, E. and Leland Jr., O.S. (1988) Angina and exertional myocardial ischemia in diabetic and nondiabetic patients: Assessment by exercise thallium scintigraphy. Annals of Internal Medicine, 108, 170-175.

[13] Deedwania, P.C. and Carbajal, E.V. (1991) Silent myocardial ischemia: A clinical perspective. Archives of Internal Medicine, 151, 2373-2382. doi:10.1001/archinte.1991.00400120019004

[14] Deanfield, J.E., Shea, M., Ribiero, P., de Landsheere, C.M., Wilson, R.A., Horlock, P. and Selwyn, A.P. (1984) Transient ST-segment depression as a marker of myocardial ischemia during daily life. American Journal of Cardiology, 54, 1195-1200. doi:10.1016/S0002-9149(84)80066-1

[15] Ohwada, R., Hotta, M., Kimura, H., Takagi, S., Matsuda, N., Nomura, K. and Takano, K. (2005) Ampulla cardiomyopathy after hypoglycemia in three young female patients with anorexia nervosa. Internal Medicine Journal, 44, 228-233. doi:10.2169/internalmedicine.44.228

[16] Abel, E.D. (2004) Glucose transport in the heart. Frontiers in Bioscience, 9, 201-215. doi:10.2741/1216

[17] Komniski, M.S. (2011) Interventricular heterogeneity in rat heart responses to hypoxia: The tuning of glucose metabolism, ion gradients, and function. Heart and Circulatory Physiology: American Journal of Physiology, 300, H1645-H1652.

[18] Liang, G.Y. (2008) Cardiac glucose uptake and suppressed expression/translocation of myocardium glucose transport-4 in dogs undergoing ischemia-reperfusion. Experimental Biology and Medicine, 233, 1142-1148.

[19] Davey, K.A. (2007) Immunogold labeling study of the distribution of GLUT-1 and GLUT-4 in cardiac tissue following stimulation by insulin or ischemia. Heart and Circulatory Physiology: American Journal of Physiology, 292, H2009-H2019.
[20] Domenighetti, A.A., Danes, V.R., Curl, C.L., Favaloro, J.M., Proietto, J. and Delbridge, L.M. (2010) Targeted GLUT-4 deficiency in the heart induces cardiomyocyte hypertrophy and impaired contractility linked with $\mathrm{Ca}(2+)$ and proton flux dysregulation. Journal of Molecular and Cellular Cardiology, 48, 663-672. doi:10.1016/j.yjmcc.2009.11.017

[21] Das, U.N. (2003) Insulin: An endogenous cardioprotector. Current Opinion in Critical Care, 9, 375-383. doi:10.1097/00075198-200310000-00007

[22] von Lewinski, D. (2010) Functional effects of glucose transporters in human ventricular myocardium. European Journal of Heart Failure, 12, 106-113.

[23] Stanley, W.C., Lopaschuk, G.D. and McCormack, J.G. (1997) Regulation of energy substrate metabolism in the diabetic heart. Cardiovascular Research, 34, 25-33. doi:10.1016/S0008-6363(97)00047-3

[24] Carley, A.N. and Severson, D.L. (2005) Fatty acid metabolism is enhanced in type 2 diabetic hearts. Biochimica et Biophysica Acta, 1734, 112-126. doi:10.1016/j.bbalip.2005.03.005

[25] Boudina, S. and Abel, E.D. (2007) Diabetic cardiomyopathy revisited. Circulation, 115, 3213-3223. doi:10.1161/CIRCULATIONAHA.106.679597

[26] Lopaschuk, G.D., Lakey, J.R., Barr, R., Wambolt, R., Thomson, A.B., Clandinin, M.T. and Rajotte, R.V. (1993) Islet transplantation improves glucose oxidation and mechanical function in diabetic rat hearts. Canadian Journal of Physiology and Pharmacology, 71, 896-903. doi:10.1139/y93-136

[27] Desouza, C., Salazar, H., Cheong, B., Murgo, J. and Fonseca, V. (2003) Association of hypoglycemia and cardiac ischemia: A study based on continuous monitoring. Diabetes Care, 26, 1485-1489. doi:10.2337/diacare.26.5.1485 